\title{
PREFACE
}

\section{Updates in Phonological Intervention}

I am extremely pleased to offer readers of Seminars in Speech and Language this very timely and innovative issue that provides updates on phonological intervention. The tireless and capable guest editor for this volume is Shelley Velleman, who has gathered together some of the most talented clinical researchers working with childhood phonological disability today.

The challenge of working with children who demonstrate significantly delayed or disordered phonological development in the absence of known etiology continues to be substantial. Furthermore, in today's climate of eligibility requirements and managed care, ever more emphasis is placed on helping such children achieve functional and intelligible speech most efficaciously. The majority of the articles in this volume concern themselves directly with the principles of assessment, description, readiness for therapy, and intervention techniques that hold the potential to improve the effectiveness and efficacy of work with such children.

There were a number of possible ways to arrange the contributions, but we have decided to present them in the order in which clinical decision making most typically occurs. The first article, "Pattern-based approaches to phonological therapy," by Stoel-Gammon, Stone-Goldman, and Glaspey, begins with the process of improving the description of children's phonological abilities and needs by reviewing and extending the use of phonological process analysis in the assessment, therapeutic planning, and intervention options for children with multiple misarticulations. As with each of the other con- tributors, Stoel-Gammon et al progress from a theoretical model of the value of such pattern analysis to a clear illustration of how application of the approach was utilized to improve the phonological skills of an actual child seen for therapy.

The second contribution, by Karen Forrest, addresses the question, "Are oral-motor exercises useful in the treatment of phonological/ articulatory disorders?" This article, together with the next contribution, "From phonological therapy to phonological awareness" by Joy Stackhouse, Bill Wells, Michelle Pascoe, and Rachel Rees, considers, in part, what skills may make a child ready for direct work on phonological targets. Forrest reviews what we currently know about the role of oral-motor work in facilitating phonological change. Although cautious about the ability of future research to provide evidence of the utility of oral-motor exercises in the treatment of children with phonological and articulatory disorders, Forrest concludes that little, if any, research currently supports the legitimacy of using such procedures to facilitate phonological development, particularly when alternative direct methods of working on speech sound production do appear to produce therapeutic gains. When clinicians implement those therapy approaches and components with the highest degree of researched efficacy, they make better use of scarce therapy time.

A similar discussion is raised in portions of the chapter contributed by Stackhouse et al. The role of phonological awareness in the child's readiness to participate in and profit

Updates in Phonological Intervention; Editors in Chief, Nancy Helm-Estabrooks, Sc.D., and Nan Bernstein Ratner, Ed.D.; Guest Editor, Shelley Velleman, Ph.D. Seminars in Speech and Language, volume 23, number 1, 2002. ${ }^{1}$ Department of Hearing and Speech Sciences, University of Maryland, College Park, Maryland. Copyright (C 2002 by Thieme Medical Publishers, Inc., 333 Seventh Avenue, New York, NY 10001, USA. Tel: +1(212) 584-4662.0734-0478,p;2002,23;01, 001,002,ftx,en;ss100106x. 
from phonological intervention is carefully examined, with practical examples from the types of interactions most often seen during therapy. Most important, Stackhouse et al analyze the metaphonological demands of therapy participation often taken for granted and discuss the need to carry out therapy task analysis and assess children's metaphonological abilities before embarking on many of the contemporary approaches to phonological intervention.

Velleman discusses the role of "Phonotactic therapy" in readying the highly unintelligible child with phonotactic limitations for therapy specifically directed at segmental accuracy. When children cannot duplicate the patterns of syllable and word shapes characteristic of the ambient language, a broader perspective must be taken to induce adult-like frames before the accuracy of individual segmental targets can be addressed. Velleman provides a case study to illustrate how a phonotactic approach was used to expand greatly one child's use of syllable and word shapes in preparation for work on misarticulated segmental targets.

The next two contributions offer specific clinical treatment options for improving the realization of segmental targets in the speech of phonologically impaired children. Barlow and Geirut discuss "Minimal pair approaches to phonological remediation." Because minimal pair therapy addresses the phonemic contrasts of the target language, it is inherently meaning based, as are a number of the approaches discussed in this volume. Given the increasing evidence for the efficacy of using minimal pairs as a therapy construct, they turn specifically to the relative benefits of various types of potential contrasts in inducing maximum generalization. It is important to note that some of the pairings that appear to induce greatest progress are not necessarily selfevident or consistent with many older recommendations for ordering therapy targets.

Finally, Tyler turns her attention to "Language-based intervention for phonological disorders." Although the approach is not appropriate for all children, Tyler finds clear evidence of its utility with children having concomitant phonological and language problems and additionally provides suggestions for predicting which of such children are most likely to make greater or lesser gains in phonology from such an approach. Beyond its use with children having specific profiles of concomitant impairment, Tyler also notes the utility of language-based intervention when service delivery options are not conducive to other phonological intervention approaches.

Taken together, this volume offers contemporary, evidence-based suggestions that can improve the effectiveness of your therapy with phonologically disordered children. All of the contributors have provided a clear rationale for their therapy approaches and given clear examples of their work "in action" with an actual challenging case. We trust that you will find great satisfaction in assessing the effectiveness of their recommendations with the children on your caseload.

Nan Bernstein Ratner Co-Editor in Chief ${ }^{1}$ 